\title{
Autoconcepto y ansiedad: detección de indicadores que permitan predecir el riesgo de padecer adicción a la actividad física
}

\section{Self-concept and anxiety: detection of indicators allowing to predict addiction risk to physical activity}

\section{Autoconceito e ansiedade: detençáo de indicadores que permitam predizer o risco de padecer adiçáo à atividade física}

David Molero López-Barajas, Rosario Castro-López, Ma Luisa Zagalaz-Sánchez

Universidad de Jaén - España

Resumen: El propósito del estudio es determinar el número de participantes afectados de adicción al ejercicio, asociar indicadores sociodemográficos a niveles elevados de vigorexia y hallar variables predictoras que permitan explicar un porcentaje de la variable de estudio. Los participantes son 154 deportistas (edad media 24.97 años, \pm 6.90 ), distribuidos en dos grupos de edad (menores de 30 y mayores de 30 ańos) y cuatro localidades de distinto número de habitantes. Los instrumentos empleados son la Escala Adonis, para evaluar vigorexia, el cuestionario de Autoconcepto Forma 5 (AF5) y la escala STAI de ansiedad estado/rasgo. Un alto porcentaje de los sujetos de la muestra $(62.8 \%)$ presentan un grado moderado de preocupación patológica. Se han encontrado diferencias significativas en las puntuaciones de vigorexia en función de las variables edad, experiencias previas negativas, localidad de residencia y número de horas de práctica deportiva. Asimismo, a través de un estudio de regresión múltiple, se han determinado las variables que mejor predicen la adicción al ejercicio (Ansiedad Estado, Horas semanales en el gimnasio y dimensión Académico-laboral del AF5).

Palabras clave: autoconcepto, ansiedad, vigorexia, actividad física, promoción de la salud.

Abstract: The purpose of the study is to determine the number of participants affected exercise addiction, to relate sociodemographic indicators to high levels of reverser anorexia, and to find predictive variables to explain a percentage of the variable under examination. The participants are 154 bodybuilders (mean age 24.97 years, \pm 6.90 ), divided into two age groups (under 30 and over 30 years) and four locations in different numbers of inhabitants. The instruments used are the Adonis Scale to assess reverse anorexia, the questionnaire of self-concept form 5 (AF5) and state/trait anxiety scale (STAI). A high percentage of subjects in the sample $(62.8 \%)$ show moderate pathological concern. We have found significant differences in scores of reverse anorexia depending on the variable of age, previous negative experiences, place of residence, and number of hours devoted to exercising. Also, through a multiple regression study, we have determined the variables that best predict exercise addiction (Anxiety State, weekly hours in the gym and Academic-labor dimension AF5).

Keywords: self-concept, anxiety, reverse anorexia, physical activity, health promotion.

RESUMO

O propósito desse estudo é de determinar o número de participantes afetados à adição ao exercício, associar os indicadores sociodemográficos aos níveis de vigorexia e encontrar variáveis que predigam e que permitam explicar a porcentagem da variável do estudo. Os participantes foram 154 esportistas (idade media 24.97 anos, \pm 6.90 ), distribuídos em dois grupos de idade (menores de 30 anos e maiores de 30 anos) e quatro localidades com distintos números de habitantes. Os instrumentos aplicados foram a Escala de Adonis, para avaliar a vigorexia, o questionário de Autoconceito Forma 5 (AF5) e a Escala de STAI de ansiedade estado/traço. Uma porcentagem dos sujeitos da mostra (62.8\%) apresentou um grau moderado de preocupaçáo patológica. Foram encontradas diferenças significativas nas pontuaçôes de vigorexia em funçâo das variáveis de idade, experiência negativa prévia, localidade de residência e número de horas de prática esportiva. Com o estudo de regressáo múltipla, foram determinadas as variáveis que podem predizer melhor a adição ao exercício (ansiedade estada, horas semanal na academia e dimensão acadêmica- laboral de AF5).

PALAVRAS CHAVE: autoconceito, ansiedade, vigorexia, atividade física, promoção da saúde.

\section{Introducción}

Son distintos los aspectos relacionados con la vigorexia (reverse anorexia), patología psicológica que ha ido cobrando protagonismo en los últimos años debido a la cada vez más creciente importancia del aspecto físico. Entre ellos el aspecto

Correspondencia: David Molero López-Barajas, Universidad de Jaén Campus Las Lagunillas, Edificio C5, -23071- Jaén. España E-mail: dmolero@ujaen.es.Teléfono: (0034)953213436 físico, como apariencia, es el conjunto de características físicas y estéticas que nos hacen ser percibidos diferentes unos de otros y que resulta externo a la autopercepción. Por su parte, la imagen corporal es la representación del cuerpo que cada persona construye en su mente. Esta doble visión del sujeto en la sociedad, tanto interna (autopercepción) como externa, no necesariamente están enlazadas y correlacionan de acuerdo a la realidad. En ocasiones se produce una discordancia 
entre ambas puesto que personas cuya apariencia física se aleja de los cánones de belleza estereotipados por la sociedad actual tienen una imagen corporal adecuada y/o satisfactoria. Del mismo modo, personas con apariencia física dentro del estereotipo "perfecto", o socialmente deseable, perciben una imagen corporal totalmente distinta a la real, llegando a producir sesgos en su autopercepción (Raich, 2000).

La importancia de la apariencia física y de la imagen corporal es un aspecto que ha ido cobrando protagonismo con el paso del tiempo. Esto se debe fundamentalmente a la sociedad actual, globalizada y manejada por los medios de comunicación que muestran constantemente cuerpos esculturales y figuras impecables. Esta sobredimensión de lo físico implica en algunos ámbitos la exclusión por el mero hecho de no pertenecer a ese rango estereotipado, lo que puede llevar al ciudadano a una preocupación moderada, en ocasiones grave, hasta el punto de modificar su propio esquema corporal y, por consiguiente alterar dicha percepción; así como la importancia de las influencias sociales positivas para aquellos que se divierten con la práctica de la actividad física (Moreno, Hernández y González-Cutre, 2009) en especial durante la adolescencia. Distintos estudios han abordado esta temática y su relación con el autoconcepto físico en nuestro contexto analizando estos aspectos en distintos grupos de edad (Contreras, Fernández, García, Palau y Ponseti, 2010; Esnaola, Goñi y Madariaga, 2008; Gallo, González y Salinero, 2010; Gońi, Rodríguez y Esnaola, 2010; Infante y Gońi, 2009; Moreno, Cervelló y Moreno, 2008).

El término empleado para hacer referencia a la alteración de la imagen corporal ha cambiado históricamente desde que a finales del siglo XIX, Enrico Morselli (1852-1929), propuso el término "dismorfofobia" (miedo a la propia forma), posteriormente, en 1903, Janet lo denomina como "la obsesión por la vergüenza del propio cuerpo" y actualmente es conocido como Trastorno Dismórfico Corporal (Raich, 2000).

Garner y Garfinkel (1981), en relación con la evaluación de la imagen corporal, ponen de manifiesto que la alteración de la misma puede darse en dos dimensiones. En primer lugar, los mencionados autores identifican una alteración perceptual: referida a la dificultad o imposibilidad que la persona manifiesta para estimar su tamaño y forma corporal. En segundo lugar existiría una alteración cognitivo-afectiva: referida a la existencia de pensamientos y sensaciones negativas a causa de su apariencia física. Esta doble perspectiva resulta de utilidad para determinar cuál es el aspecto de la imagen corporal que se manifiesta alterado, puesto que no tienen porque caminar en comunión. Tal es la independencia de ambos enfoques, que autores como Thompson (1990) o Vaz, Peńas y Ramos (1999), sugieren que no se confundan "alteración de la imagen corporal" con "insatisfacción corporal"; ya que el primero engloba al segundo entre otros aspectos.

El origen del concepto Vigorexia (reverse anorexia) se atri- bute Pope, Katz y Hudson (1993), quienes en el Laboratorio de Psiquiatría Biológica del Hospital McLean, descubrieron este trastorno. Al estudiar el uso de esteroides anabolizantes en varones reclutados en gimnasios de halterofilia de Boston y se encontró que un gran número de ellos se percibían como pequeños y flacos, cuando de hecho eran grandes y musculosos (Pope y Katz, 1994). Este fenómeno fue denominado inicialmente como anorexia nerviosa inversa: "reverse anorexia" puesto que compartía características similares a la anorexia nerviosa (como la distorsión de la imagen corporal y la búsqueda incesante del cuerpo deseado aun incurriendo en conductas de riesgo para su propia salud) pero con síntomas inversos a ésta (Pope, Katz y Hudson, 1993).

Varios han sido los estudios que desde entonces Pope ha llevado a cabo, variando este término inicial y popularizando la denominación de "Complejo de Adonis" (Pope, 2000), que ha utilizado para titular sus trabajos. Según la mitología griega este concepto hace referencia a un hombre extremadamente atractivo, como símbolo de belleza masculina. Entre las evidencias de sus trabajos hemos de hacer mención a la afirmación de que de los 9 millones de usuarios de gimnasios de EE.UU, algo más del $10 \%$ podría estar afectado de este trastorno. Esta cifra varía mucho en función del estado o país en el que se pretenda estudiar, ya que en otra de sus investigaciones ratificaron como los hombres de países del este gozaban de una menor insatisfacción corporal que los hombres occidentales (Jeffrey, Gray y Pope, 2005).

Otros autores (Muñoz y Martínez, 2007) han ido matizando el término, como un trastorno somatomorfo, sugiriendo también términos como dismorfia muscular o vigorexia, por lo que se sitúa al lector en un campo un tanto difuso en el que coexisten diferentes nomenclaturas, aunque éste no es uno de los aspectos que más controversia produce, ya que el problema no es ponerle nombre sino determinar de qué se trata. A continuación se aborda el concepto de este trastorno, adoptando a partir de este momento el término vigorexia, para evitar posibles confusiones terminológicas.

La vigorexia puede definirse como un trastorno que se caracteriza por una preocupación extrema por verse con poca masa muscular, cuando en realidad se es musculoso, renunciando a oportunidades sociales y ocupacionales a causa de la necesidad de ejercitarse y evitando situaciones donde el cuerpo pueda ser visto en público. Pope, Katz y Hudson (1993) lo definen como un trastorno mental caracterizado por una obsesión exagerada por ganar masa muscular y perder grasa.

Habitualmente, es común que las personas vigoréxicas pasen muchas horas en el gimnasio, muestren alteraciones en sus hábitos de alimentación, consumiendo exageradamente suplementos proteicos con componentes quemadores de grasas y/o abusen de sustancias, principalmente esteroides anabolizantes para lograr de manera rápida un mayor desarrollo muscular, con la posibilidad de múltiples complicaciones mé- 
dicas e incluso letales (Pérez, Nelly, Rodríguez y Gempeler, 2007).

Esta preocupación obsesiva por la figura y búsqueda incesante de la belleza física acompaña a una distorsión del esquema corporal, llevándolos a una continua práctica deportiva sin límites, sin considerar los riesgos que conlleva para su salud. En este sentido, Vives y Garcés (2003) señalan como características definitorias que caracterizan este síndrome psicopatológico las siguientes: Necesidad de acudir al gimnasio o realizar actividad física, ritual que alcanza varias horas diarias; Sensación de culpa si se produce un fallo en la rutina de práctica deportiva; Objetivo de aumentar hasta el infinito su masa muscular (nunca llega a ser suficiente); Pensamientos de cuerpo perfecto como el que tiene el mayor músculo posible; El deporte se convierte en su vida sin límites; La alteración de la imagen corporal es el componente principal; Obsesión con la mejora de ciertos defectos físicos, no se sienten a gusto con su propio cuerpo; Dieta estricta que suele consistir en una ingestión masiva de proteínas e hidratos de carbono y total ausencia de grasa; Baja autoestima, poca madurez y pensamientos de que tienen poco atractivo entre el sexo opuesto debido a su aspecto físico; Exigentes consigo mismos, perfeccionistas, introvertidos y extremistas; Presentación de síntomas de otras alteraciones psicológicas como ansiedad, depresión y trastornos obsesivos-compulsivos.

En cuanto a la dieta que llevan a cabo estos sujetos, distintos autores coinciden en su carácter estricto (Vives y Garcés, 2003; Baile, 2005), aunque Vives y Garcés (2003) hacen hincapié en la ingestión masiva de proteínas e hidratos de carbono y total ausencia de grasas, mientras que Baile (2005) destaca el consumo de sustancias peligrosas para el desarrollo muscular, tales como hormonas o esteroides anabolizantes.

Continuando con el estudio del término vigorexia así como de sus características y manifestaciones principales, a continuación realizaremos una aproximación diferencial a la misma, es decir intentaremos caracterizar qué no es la vigorexia.

Algunos estudios relacionan los comportamientos de tipo vigoréxico con las adicciones no químicas como juego, poder, dinero, trabajo, comida, sexo o deporte. La práctica del ejercicio físico provoca la liberación de opiáceos endógenos, como la endorfina, generando una sensación de placer natural; se ha demostrado que este tipo de sustancias combaten el estrés y mejoran la inmunidad (Pacheco, Serrano y Echegaray, 2007). Estas hormonas fisiológicas que ocasionan analgesia y una sensación de felicidad y relajación se liberan a la circulación cuando se alcanza el $76 \%$ de la frecuencia cardiaca máxima de entrenamiento. Este hecho tiene como fin contrarrestar el dolor o la sensación de cansancio extremo que el ejercicio físico y prolongado produce en el deportista. Todo esto sin sumarle los sentimientos negativos que experimentan los adictos al deporte cuando no pueden practicarlo, consi- derándolo como un sentimiento similar al de la abstinencia.

Quizás esta sea una de las explicaciones que nos lleve a pensar que se trata de una adicción al ejercicio físico, puesto que cuanto más ejercicio físico realizan, mayor es la cantidad de endorfinas que liberan a sangre y, por tanto, mejor se sienten. Pero no sólo existen aspectos similares entre Vigorexia y Adicción, ya que los vigoréxicos muestran diferencias con respecto a los adictos al deporte. El adicto a la práctica deportiva realiza ejercicio físico buscando disminuir ese estado previo desagradable (abstinencia), pero también consigue un estado interno agradable, por lo que se produce en este caso un reforzamiento positivo. Aspecto que no ocurre en el sujeto vigoréxico ya que realmente no disfrutan haciendo ejercicio físico sino que solo evita el malestar que le produce no hacerlo; esto significa que obtiene un reforzamiento negativo que le lleva a la realización de nuevo de esta conducta reiterada del ejercicio.

También merece especial atención el Trastorno Dismórfico Corporal (TDC). Este trastorno se caracteriza por una preocupación excesiva y patológica por un defecto imaginado del cuerpo o la apariencia, o si realmente existe, por una reacción excesiva hacia él. La vigorexia en este caso, si ha sido propuesta como una variable del TDC, ya que los sujetos manifiestan como síntoma central ver su cuerpo pequeño y débil (Lantz, Rhea y Cornelius, 2002; Ramos, Pérez de Eulate, Liberal y Latorre, 2003). Esta manifestación afecta a la vida del individuo ocasionando malestar y alterando sus relaciones sociales, laborales, familiares, etc.

En la revisión de la cuarta edición del manual de diagnostico DSM IV-R, en su versión en lengua española (LópezIbor, 2009), y con respecto a los criterios diagnósticos del TDC, aparecen como síntomas y trastornos asociados, los problemas con la visión de la masa muscular que padecen ciertos deportistas, hecho que no aparecía en revisiones anteriores y que nos conduce a la hipótesis de que la vigorexia pueda ser diagnosticada como un TDC. A veces se habla de trastorno dismórfico muscular (TDM) como un subtipo de TDC, el cual podríamos considerarlo como sinónimo de vigorexia, por lo que se podría concluir afirmando que la vigorexia es un TDC y más concretamente, un TDM.

Es cierto que la vigorexia se ha propuesto en ocasiones como un Trastorno de la Conducta Alimentaria (TCA), pues quienes la padecen comparten la preocupación desmedida por su aspecto físico, la distorsión del esquema corporal, la obsesión por pesar los alimentos, el interés por las comidas, calorías y composición de lo que se ingiere, la auto-observación, pesarse varias veces al día, la supresión de grasas y otros (De la Serna, 2004). Sin embargo no se ha comprobado que los sujetos vigoréxicos tengan una conducta alimentaria patológica y ningún autor ha considerado este hecho como elemento definitorio.

En ocasiones, los sujetos vigoréxicos tienden a una restric- 
ción alimentaria y al uso de fármacos (como suplementos proteicos y abuso de esteroides anabolizantes) para incrementar su masa muscular. Este hecho nos lleva a la existencia de todo un mundo de productos alimenticios, complejos vitamínicos, sustancias orgánicas y sintéticas, denominadas también ergogénicas o que ayudan a crecer, que rodean el mundo de los gimnasios. Obviamente, la ingesta de estos productos acarrea unos efectos negativos para la propia salud del deportista. En un reciente estudio (Jenaro, Flores, Bermejo y Cruz, 2011) se presenta un recurso (Cuestionario de Evaluación de la Imagen Corporal) para evaluar la imagen corporal para la detección temprana de trastornos de la conducta alimentaria, siendo de utilidad para intervenir de manera precoz en los casos descritos.

Tras realizar estas precisiones conceptuales, el problema de nuestro estudio pretende dar respuesta a si es posible conocer el autoconcepto, quiénes sufren vigorexia y ansiedad entre los participantes del estudio, todo ello medido a través de distintos instrumentos estandarizados (ADONIS, STAI, AF5) y a su vez si se puede establecer cuáles son las variables predictoras de éstos aspectos en nuestro contexto.

De manera global, en función del problema considerado, presentamos los objetivos del estudio: (a) Determinar cuál es el número de personas afectadas de este trastorno (o que presenten manifestaciones potencialmente patológicas) en la muestra. (b) Asociar indicadores sociodemográficos y personales de la muestra a niveles elevados de vigorexia. (c) Hallar variables predictoras, a través del estudio de aspectos psicológicos del deportista que permitan explicar un porcentaje de la variable de estudio.

\section{Método}

\section{Participantes}

Los participantes son 154 deportistas $(\mathrm{n}=154), 142$ son hombres (92.21\%) y 12 mujeres (7.79\%), con edades comprendidas entre los 16 y 49 años (edad media de 24.97 años, desviación típica $= \pm 6.90$ ), distribuidos en dos grupos de edad, 129 sujetos menores de 30 años $(83.8 \%)$ y 25 participantes mayores de 30 años (16.2\%).

Los sujetos realizan habitualmente trabajo de musculación en gimnasios de la provincia de Jaén. Para ello se estimó conveniente encuestar a usuarios de gimnasios de las dos ciudades más grandes de dicha provincia, avalados según el Instituto Nacional de Estadística (INE) como las dos únicas que cuentan con más de 50.000 habitantes, Jaén (116790 habitantes) y Linares (62347 habitantes). Por otra parte, se consideró oportuno analizar las mismas cuestiones en dos núcleos rurales, aportando a este mismo estudio datos de dos localidades con poblaciones comprendidas entre los 5.00010.0000 habitantes, Mengíbar (9572 habitantes) y Arjona
(5802 habitantes). En la distribución de la muestra por localidades se respetaron los criterios de proporcionalidad en función del número de habitantes en los censos oficiales de los mismos; de esta manera, se seleccionaron 88 sujetos de la ciudad de Jaén (57.14\%), 46 participantes de Linares (29.87\%), 12 sujetos de Mengíbar (7.79\%) y 8 (5.19\%) de Arjona.

\section{Instrumentos}

Además de los instrumentos empleados se ha recogido información adicional, incluyéndose una serie de cuestiones que contenían algunas variables personales y sociodemográficas de los participantes, tales como edad (menos de 30 años y mayores de 30 años), sexo (hombre o mujer), ciudad de residencia (Jaén, Linares, Mengíbar y Arjona), número de horas dedicadas semanalmente al gimnasio (1 a 5, 6 a 10, 11 a 15, 16 a 20 y más de 20 horas) y el hecho de haber sufrido alguna experiencia negativa a causa de su apariencia física (sí o no). $\mathrm{Su}$ finalidad ha sido asociar el perfil sociodemográfico con puntuaciones elevadas en vigorexia entendiéndose como una aproximación personal a dicho perfil sin abordar con profundidad los detalles de estos aspectos sociodemográficos.

\section{Cuestionario Complejo de Adonis}

El primer instrumento considerado ha sido el cuestionario denominado Complejo de Adonis propuesto por Pope, Katherine, Phillips y Olivardia (2003), traducido y validado al español por Baile, Monroy y Garay (2005). Dicho cuestionario consta de 13 ítems con 3 opciones de respuesta y valora el grado de preocupación que un sujeto siente por su apariencia física y hasta qué punto puede influir negativamente a los demás aspectos de su vida, pasando a ser dichas conductas patológicas. La puntuación máxima de la prueba es de 39 puntos. Puntuaciones inferiores a 9 puntos informan de una ausencia de preocupación patología, entre 10 y 19 puntos existe un grado moderado, entre 20 y 29 puntos se padece un Complejo de Adonis serio y por último, puntuaciones entre 30 y 39 informan de un problema muy serio con la imagen corporal. La fiabilidad de la escala (consistencia interna) es de .70.

\section{Autoconcepto Forma 5 (AF5)}

El cuestionario Autoconcepto Forma 5 -AF5- (García y Musitu, 1999) surge como versión ampliada y actualizada de otra ya existente, el autoconcepto forma A o AFA. En sus 30 elementos pretende evaluar en el sujeto 5 dimensiones básicas del autoconcepto: académico-laboral (11 ítems), social (5 ítems), emocional (9 ítems), familiar y física (6 ítems). Esta última, permite recoger al evaluador información adicional y sin duda muy útil en cuanto a la manera en la que el sujeto se autopercibe. Los índices de fiabilidad de la escala, alfa de Cronbach, varían entre .71 y .84 . 


\section{Ansiedad Estado-Rasgo (STAI)}

El cuestionario STAI - State Trait Anxiety Inventory- (Spielberger, Gorsuch y Lushene, 1970), traducido y validado al español en 1999 (Spielberger, Gorsuch y Lushene, 1999), permite diferenciar dos escalas de autoevaluación de ansiedad, como estado (E) y como rasgo (R). La sub-escala Ansiedad Estado (A/E) posee 20 ítems, en los que el sujeto describe cómo se siente "en un momento particular", teniendo cuatro opciones de respuesta en función del grado de acuerdo al enunciado de cada cuestión; mientras que en la dimensión Ansiedad como Rasgo (A/R), también con 20 cuestiones, el sujeto puede manifestar como se siente "generalmente". Aunque originalmente fue ideado como instrumento para analizar la ansiedad en adultos sin alteraciones psiquiátricas, el STAI ha mostrado ser útil para medir ambos conceptos tanto en escolares de estudios medios y superiores como en pacientes de diversos grupos clínicos. Los coeficientes alfa de consistencia interna informados para esta prueba por los autores de la misma en la A/E muestran un .83 en hombres y .86 en mujeres. Con respecto a la $A / R$, se obtuvo un coeficiente alfa de Cronbach de .86 en varones y .86 en mujeres, valores moderadamente altos que respaldan la fiabilidad del instrumento.

\section{Procedimiento}

Para la aplicación de los instrumentos detallados se llevó a cabo una interacción con el monitor o encargado de cada uno de los gimnasios elegidos, fundamentalmente a través de charlas, con el propósito de que sea una persona de su entorno y confianza la que transmitiera las ideas fundamentales del interés y contenido del proyecto. En cada coloquio con el profesional encargado se hizo especial hincapié en la importancia que conlleva un estudio en dicho ámbito (hasta el momento no estudiado en la provincia) y en la facilidad de la cumplimentación de las pruebas. Asimismo se expuso la idea del carácter voluntario y anónimo que este presentaba con el propósito de que intentaran animar a los usuarios de gimnasios a que el nivel de participación fuera óptimo.

Se añadieron facilidades para los participantes indicándoles que cumplimentaran los instrumentos en su domicilio en un ambiente más favorecedor y abierto a la comprensión, dado que un ambiente óptimo aumentaría la capacidad de entendimiento y con ella su sinceridad, desembocando de esta manera en un aumento de la calidad de los resultados. Los sujetos desconocían el propósito del estudio con el fin conseguir sinceridad en las respuestas y reducir el efecto de la deseabilidad social, en la medida de lo posible. En el momento de la recogida de los cuestionarios se verificó la correcta y completa contestación de los mismos.

\section{Análisis estadístico}

La realización de los correspondientes análisis estadísticos se basaron en el cálculo de los estadísticos descriptivos (medias y desviaciones típicas), pruebas de diferencia de medias en aquellas variables con dos alternativas de respuesta, análisis de varianza (ANOVA) para los casos en donde existían tres o más alternativas de respuesta, para establecer la existencia de diferencias significativas entres las distintas variables sociodemográficas y la valoración de la anorexia. Asimismo, se realizó un análisis de regresión múltiple por el procedimiento de pasos sucesivos (stepwise) para conocer las variables que explicaban un mayor porcentaje de la variable vigorexia. El estudio estadístico se realizó con el apoyo del software estadístico SPSS en su versión 19.0.

\section{Resultados}

\section{Valoración de la vigorexia}

Se presentan los resultados en función de los objetivos planteados en el estudio, por tanto en primer lugar informaremos, de acuerdo al primer objetivo, de los participantes que presentan manifestaciones potencialmente patológicas. En la siguiente tabla (véase Tabla 1), se pueden apreciar las puntuaciones obtenidas por la muestra en el primero de los instrumentos considerados, Escala Adonis, según las cuales podemos informar de ausencia de preocupación patología, grado moderado, grado serio y problema muy serio con la imagen corporal.

Tabla 1. Resultados de la Escala Adonis (estadísticos descriptivos)

\begin{tabular}{lccc}
\hline & Puntuación (PD) & Frecuencia & Porcentaje \\
\hline Ausencia preocupación patológica & $0-9$ & 0 & $0 \%$ \\
Grado moderado preocupación patológica & $10-19$ & 97 & $62.8 \%$ \\
Grado serio preocupación patológica & $20-29$ & 55 & $35.9 \%$ \\
Problema muy serio preocupación patológica & $30-39$ & 2 & $1.3 \%$ \\
\hline Media $=18.67$; Desviación típica=3.63 & & &
\end{tabular}

Con respecto a ésta cuestión, los resultados han sido llamativos, ya que más de un $37 \%$ de los participantes alcanzan puntuaciones superiores a 19 puntos (grado serio de patolo- gía), hecho que exhibe una grave preocupación con su imagen corporal $(M=18.6 ; D T=3.63)$. 
Diferencia entre los indicadores sociodemográficos y el nivel de Vigorexia

En relación con el segundo propósito de la investigación, asociar indicadores sociodemográficos de la muestra a niveles elevados de vigorexia, los análisis realizados indicaron diferencias de medias $(D M)$ significativas en Vigorexia en función de la Edad de los participantes $(t=2.070 \quad p<.05)$. Los menores de 30 años (Media $=22.98)$ muestran valores significativamente superiores en Vigorexia vs. a los mayores de 30 años (Media=16.62).

Por otro lado, el hecho de haber tenido o no experiencias negativas a causa del propio cuerpo (Experiencias Negativas) también influyó significativamente en la puntuación obtenida en Vigorexia. Concretamente, los participantes con experiencias negativas (Media $=20.65$ ) mostraron una puntuación media en vigorexia (Media $=18.84$ ) significativamente superior a los participantes que informaron no haber tenido dichas experiencias $(t=3.517 p<.05)$.

También se encontraron diferencias significativas en $\mathrm{Vi}$ gorexia en función de la Ciudad de residencia de los participantes, realizando para ello un ANOVA al compararse más de 2 ciudades, obteniendo los siguientes resultados: $[F(3,126)=4.205 ; p<.05]$. Para analizar entre qué localidades existían las diferencias (Jaén, Linares, Mengíbar y Arjona), se realizaron pruebas a posteriori utilizando el estadístico de Scheffè. Específicamente, la puntuación media en el Cuestionario ADONIS fue significativamente superior en la ciudad de Jaén $v s$. a la de Arjona [ $t=3.926 ; p<.05]$, así como en la de Linares $v s$. a la de Arjona $[t=3.721 ; p<.05]$. No se encontraron diferencias significativas entre las puntuaciones de Vigorexia de los restantes pares de comparaciones de las demás ciudades.

Finalmente, el ANOVA realizado entre Vigorexia y el Número de horas a la semana que los participantes dedicaban a ir al gimnasio ( 1 a 5, 6 a 10, 11 a 15, 16 a 20 y más de 20 horas), puso de manifiesto diferencias significativas $[F(4,126)=3.706$; $p<.05]$. Las comparaciones a posteriori realizadas mediante la prueba de Scheffè mostraron que las personas que asisten al gimnasio más de 20 horas a la semana muestran puntuacio- nes medias en Vigorexia significativamente superiores a todos los restantes grupos, personas que asistían de 1 a 5 horas $(t=5.010 ; p<.05)$, de 6 a 10 horas $(t=4.384 ; p<.05)$, de 11 a 15 horas $(t=4.356 ; p<.05)$ y de las personas que asistían entre 16 y 20 horas a la semana $(t=4.141 ; p<.05)$. Las diferencias entre los restantes grupos no resultaron significativas. Por el contrario, no se encontraron diferencias de medias significativas en la puntuación en Vigorexia en función de la variable Sexo $(t<1 ; p>.05 ; n s$.$) , siendo favorable ésta a los hombres$ $(M=18.69)$ vs. a las mujeres $(M=18.42)$.

\section{Análisis de regresión múltiple}

Finalmente, para responder al tercer objetivo de la investigación, hallar variables predictoras a través del estudio de aspectos psicológicos del deportista que nos permitan explicar un porcentaje de la variable de vigorexia de los participantes, hemos realizado un análisis de regresión múltiple por el procedimiento de pasos sucesivos (stepwise). Como variables predictoras se incluyeron la ansiedad estado (AE) y la ansiedad rasgo (AR) medidas a través del STAI (Spielberger, Gorsuch y Lushene, 1970), y las cinco dimensiones que recoge la escala de autoconcepto AF5 (García y Musitu, 1999): AcadémicoLaboral (AL), Social (S), Emocional (E), Familiar (F) y Físico $(\mathrm{F})$. Además de estas variables predictoras, también se introdujeron en el modelo las variables socio-demográficas y aquellas relacionadas con la práctica deportiva que habían resultado significativas en los análisis de la varianza previamente presentados: edad, número de horas semanales en el gimnasio (horas), ciudad de residencia y el hecho de haber o no tenido experiencias negativas a causa de su cuerpo (ExperN).

Los resultados de este análisis pusieron de manifiesto que el modelo que mejor explicaba la variabilidad asociada a la vigorexia para el total de la muestra era el formado por los siguientes factores: Ansiedad Estado (A/E), horas, dimensión emocional del AF5 (E), Experiencia Negativa (ExperN), Edad y la dimensión académico-laboral del AF5 (AL). En la Tabla 2 se presentan los coeficientes de regresión estandarizados para cada uno de estos factores.

Tabla 2. Coeficientes tipificados, t y significación, para cada una de las variables predictoras del modelo para el total de la muestra

\begin{tabular}{lccc}
\hline Predictores & $\beta$ (Coeficientes tipificados) & $t$ & Sig. \\
\hline Ansiedad de Estado (AE) & .247 & 3.154 & .002 \\
Horas Semanales & .252 & 3.559 & .001 \\
Autoconcepto Emocional (E) & -.231 & 2.931 & .004 \\
Experiencia Negativa (ExperN) & -.187 & 2.595 & .010 \\
Edad & -.161 & 2.280 & .024 \\
Autoconcepto Académico-Laboral (AL) & .141 & 1.991 & .048 \\
\hline
\end{tabular}

$\mathrm{r}=.549 ; \mathrm{r}^{2}=.301$ 
Como se puede observar en la tabla anterior, las variables $A n$ siedad Estado (AE), Horas en el gimnasio a la semana y la dimensión Académico-laboral del AF5 contribuyen de manera directa a predecir la puntuación obtenida en el test de Adonis, mientras que el resto de variables incluidas en el modelo (Emocional, Experiencia Negativa y Edad) se relacionan de manera inversa con la Vigorexia. Globalmente consideradas, las variables predictoras correlacionan .549 con la variable dependiente y explican un $30.1 \%$ de la variabilidad de la vigorexia, siendo el porcentaje de varianza explicada significativo $[F(6,146)=10.491 ; p<.05]$.

\section{Discusión}

El estudio ha ido dirigido a varias líneas; la primera de ellas ha sido conocer el posible porcentaje de deportistas afectados en el trastorno objeto de estudio. Con respecto a esta cuestión, los resultados han sido arrolladores, ya que llama la atención que un alto porcentaje de la muestra alcance puntuaciones superiores a 19 puntos en las Escala Adonis. Bajo este descubrimiento, se puede lanzar la hipótesis de que cada vez más la vigorexia, como una de las manifestaciones subyacentes de los TDC, está en continuo crecimiento, aspecto que incita a una difusión generalizada (empezando por los medios de comunicación) de la conceptualización de la misma, con la finalidad principal de informar o concienciar al ciudadano sobre los peligros que conlleva el lado "no-saludable" del ejercicio físico.

Estos resultados indican que la vigorexia, presentada como uno de los peligros de esta época, vive un aumento creciente, aspecto que nos invita a saber más de los perfiles que puedan resultar ser susceptible de padecerla, por lo que se ańadió a este estudio un análisis más profundo del grupo de deportistas estudiado. Estudios relacionados con este, realizados en otros países, indican que el 10\% de los usuarios de gimnasios podrían estar afectados de este trastorno (Pope, Katherine, Phillips y Olivardia, 2003), porcentaje notablemente incrementado con el resultado obtenido. Esta desigualdad podría radicar en el hecho de que ha pasado algo más de una década desde el estudio citado hasta el momento actual y que cada vez más los hombres también se encuentran inmersos en la sociedad de culto al cuerpo, no sólo las mujeres, manifestando de manera consecuente niveles de insatisfacción corporal como los expuestos.

A través del estudio de todos los parámetros sociodemográficos y personales (o psicológicos) medidos en los participantes, resaltamos el análisis de varianza resultante de la Edad de los sujetos con respecto a su nivel de vigorexia, originando unos datos claramente diferenciados. Esta distinción atribuye al grupo de menores de 30 ańos valores de vigorexia significativamente superiores a los de los mayores de 30 años, hecho que coincide con los resultados de algunos estudios sobre los desórdenes de la imagen corporal y edad (Zagalaz,
Martínez y Rodríguez, 2005), y que hace aún más fuerte la idea de que la población con edades comprendidas entre 17 y 30 años es más susceptible de padecer cualquier tipo de trastorno relacionado con la imagen corporal que los mayores de esta edad.

De la misma manera, el hecho de haber padecido cualquier experiencia negativa a causa del aspecto físico del deportista y ponerse en entredicho su masculinidad es un dato que igualmente ha resultado significativo, de acuerdo con la revisión bibliográfica previamente analizada (Pérez, Nelly-Valencia, Rodríguez y Gempeler, 2007). Los resultados obtenidos manifiestan que los participantes que han tenido alguna experiencia negativa con su cuerpo poseen niveles significativamente superiores en vigorexia que los que no han experimentado esta sensación. Teniendo en cuenta este enunciado se puede ratificar la idea de que una experiencia traumática en la infancia o pubertad, pueden ser factores contribuyentes que le dan sentido al síntoma dismórfico y a la búsqueda compensatoria de una imagen corporal que por lo menos en apariencia, no pusiera en evidencia la vulnerabilidad interna percibida.

Un aspecto que se estimó conveniente medir, fue realizar una comparación entre un núcleo urbano, las dos ciudades más grandes de la provincia de Jaén y dos núcleos rurales, las localidades de Mengíbar y Arjona. Una de las primeras comparaciones entre varias poblaciones la realizaron $\mathrm{Chi}-\mathrm{Fu}$, Yang, Gray y Pope (2005), cuando hicieron un estudio sobre los desordenes de la imagen corporal en 55 hombres de Taiwán, Estados Unidos y Europa. Los resultados demostraron que los occidentales tiene una preocupación de su imagen corporal notablemente superior que la de los orientales, mostrando los últimos unos niveles significativamente inferiores a la importancia del cuerpo, de tal manera que en el estudio incluyeron el ideal de cuerpo perfecto, retratando las revistas americanas al hombre occidental desnudo frecuentemente, mientras que las revistas orientales fotografiaron su ideal vestido, raramente sin ropa.

En el caso que nos ocupa, los resultados confirman nuestra idea inicial, ya que se obtuvieron diferencias significativas entre ambos tipos de localidades, lo que significa que, el deportista usuario de gimnasio en una ciudad de tamaño mayor, presenta niveles de preocupación corporal considerablemente superiores que quienes usan el gimnasio en localidades más pequeńas. Tal desigualdad puede radicar en muchos aspectos, uno de ellos, y quizá el más importante, podría ser la apariencia y características físicas de los modelos publicitarios como factor de influencia social mediática. Dicho influjo provoca en el deportista un exceso de ejercicio físico para alcanzar una figura ideal por una cuestión meramente estética, siendo influenciado por modelos actuales que propone la sociedad a los cuales se les vinculan valores de éxito sexual, social y profesional (Fanjul-Peyró, 2008). 
Cuando hacemos mención a dosis adecuada, nos referimos a las horas semanales que un usuario de gimnasio (que no pertenezca a ningún tipo de club o que se encuentre inmerso en competición) dedica a hacer deporte. Esta cuestión también ha sido medida en nuestro estudio, obteniéndose para nuestra premisa resultados evidentes. Los participantes que dedican más de 21 horas semanales a la práctica deportiva han mostrado niveles de preocupación de su imagen corporal significativamente superiores en vigorexia al resto de encuestados, hecho que confirma la revisión bibliográfica previamente analizada sobre el supuesto de que el perfil de la persona con vigorexia, o potencial vigoréxico, permanece un tiempo excesivo en el gimnasio realizando una práctica deportiva sin límites (Martell, 2006).

Conocer los efectos del ejercicio físico en nuestro organismo, así como ejercitarse orientada y regularmente, controlando las rutinas y entrenamientos a realizar durante la práctica deportiva y sobre todo, asesorándose por profesionales de la prescripción del ejercicio es una propuesta que se lanza con el propósito de prevenir este riesgo para la salud, puesto que numerosos estudios atribuyen a la vigorexia como uno de los problemas importantes para la salud pública mundial en las próximas décadas (Gallo, González y Salinero, 2010). Creemos por tanto, que los datos significativos resultantes de nuestro estudio podrían ser modificados a través de programas de prevención, intentando bajar este porcentaje tan elevado como el obtenido en la muestra.

Por lo expuesto, creemos que cada vez son más las personas que poseen este tipo de trastornos de la imagen corporal y tras asociar los parámetros personales medidos de los participantes a la vigorexia, no podríamos afirmar si ésta es causa o consecuencia de la existencia de estos parámetros, pero sí modificar los aspectos estudiados. Estudios similares al nuestro inciden en la importancia de la intervención psicológica para el control alimentario en jóvenes deportistas, sus conductas alimentarias y la mejora de la calidad de vida (Olmedilla y Andreu, 2002).

Una posible solución para erradicar o evitar la evidente propagación de esta problemática podría ir dirigida a varias líneas, pero sobre todo debería aportar información hacia la prevención, incluyendo evidencias sobre el modelo estético masculino actual, crítica del paradigma hiper-musculado como el más adecuado, aportando algún conocimiento fisiológico sobre desarrollo corporal masculino y, por consiguiente, del desarrollo muscular, análisis y crítica de los productos y dietas que supuestamente conducen a ese "perfeccionamiento”, así como un correcto análisis y crítica del consumo de esteroides anabolizantes (Muñoz y Martínez, 2007). Además de todas las variables sociodemográficas y culturales medidas hasta el momento que nos aportaron diferencias significativas con la variable vigorexia, para resolver el tercer objetivo planteado se midieron de manera complementaria dos parámetros psicológicos como la Ansiedad, a través del test STAI, An- siedad de Estado y la Ansiedad de Rasgo, y el Autoconcepto, aportando datos de cinco dimensiones del mismo a través del test Autoconcepto Forma 5 (AF5): Académico-Laboral, Social, Emocional, Familiar y Físico.

En lo relativo a la Ansiedad, se ha postulado la idea de que constituye una de las consecuencias psicológicas que conlleva la vigorexia (Martell, 2006). Ahora bien, teniendo en cuenta que este estudio posee un carácter correlacional, no podemos establecer una relación causa-efecto, ya que hubiera sido necesario llevar a cabo un estudio de tipo longitudinal o con grupo control. Tampoco nos permite concluir si la ansiedad o autoconcepto es causa o consecuencia de la vigorexia.

Al igual que la ansiedad, la autoestima y el autoconcepto también aparecen como una de las causas psicológicas que pueden originar el trastorno dismórfico en la bibliografía consultada (Jódar, Vives y Garcés, 2002). Sin embargo como hemos comentado no se puede ratificar el carácter causal o consecuente de estas variables psicológicas. Otros autores, en trabajos similares al nuestro (Candel, Olmedilla y Blas, 2008) han analizado las relaciones existentes entre la práctica deportiva, el autoconcepto (también medido con el AF5), la ansiedad (evaluada con el STAI) y la depresión (medido con el BDI) en población adolescente, en concreto en chicas; demostrando en su estudio que los sujetos que practican algún tipo de actividad deportiva obtienen valoraciones más elevadas en autoconcepto (salvo en el emocional) y niveles inferiores en ansiedad y en depresión que las adolescentes que no realizan actividad física.

Con la finalidad de predecir la variable dependiente del estudio, es decir, la vigorexia, se realizó un análisis de regresión múltiple; obteniéndose unos resultados interesantes, poniendo de manifiesto que el modelo que mejor explicaba la variabilidad asociada a la vigorexia era el formado por los siguientes factores: Ansiedad de Estado (AE), Horas semanales $(\mathrm{H})$, Autoconcepto Emocional (E), Experiencia Negativa $(\operatorname{ExpN})$, Edad y Autoconcepto Académico-Laboral (AL). Como se expresó en los resultados, hemos hallado variables que contribuyen de manera directa a predecir la puntuación obtenida en el test de Adonis, como es el caso de las variables Ansiedad Estado (AE), Horas Semanales y Autoconcepto Académico-Laboral (AL), lo cual no implica que cuanto mayor sea el nivel de estas variables, más alta será la puntuación obtenida en la Escala de Adonis.

De acuerdo a lo estudiado en la revisión bibliográfica, la Ansiedad y el Número de horas dedicadas a hacer ejercicio, en nuestro caso de musculación, contribuyen de manera directa a la variable dependiente y correlaciona positivamente con la misma (Martell, 2006), hecho que se corrobora con nuestros resultados. Sin embargo, también se obtuvo una correlación positiva entre la dimensión AL del autoconcepto y la variable de estudio. Sobre este aspecto, nos hemos generado una pequeńa controversia, ya que la literatura califica el perfil vi- 
goréxico como una persona con la autoestima afectada (Muñoz y Martínez, 2007) y otros lo seńalan con baja autoestima (Mirella, 2008). Sobre esta discrepancia se han de esclarecer ciertos conceptos, el autoconcepto es una percepción que se posee de sí mismo y la autoestima está formada por la relación entre la percepción o autoconcepto y su ideal.

El resultado obtenido en este estudio sobre la correlación positiva entre Autoconcepto AL y vigorexia, por tanto, lejos de generar una disputa, aclara aun más las manifestaciones básicas de este trastorno, ya que el sujeto no tiene porqué verse mal a sí mismo (autoconcepto) sino que en función de su ideal, o de lo que le gustaría ser, realiza un juicio negativo (autoestima). El hecho de que los participantes gocen de un autoconcepto adecuado, hace referencia al conocimiento que alguien tiene de sí mismo. Se sabe que estas personas pasan muchas horas en el gimnasio y privan mucho de su alimentación los nutrientes grasos, por lo que tampoco nos resulta muy extrańo el hecho de que se perciban de manera adecuada. Ahora sí, cuando se habla de autoestima se hace referencia al valor que la persona se atribuye a sí misma, o lo que es lo mismo, a la relación entre como se ve, y como le gustaría verse; el que esta diferencia sea mayor o menor determinará el nivel de autoestima y esto nos lleva a replantearnos ciertas manifestaciones características de este trastorno.

De la misma manera que las variables AE, Horas y $\mathrm{AL}$ contribuyeron de manera directa en el análisis de regresión, el resto de variables incluidas en el modelo (Autoconcepto E, ExpN y Edad) se relacionan de manera inversa con la vigorexia. Esto significa que cuanto menor sea el valor de estas variables, mayor será la puntuación obtenida en vigorexia. Los resultados que arrojan estas variables, coinciden y ratifican las evidencias halladas en otros estudios; Jódar, Vives y Garcés (2002) los califican como poco maduros, premisa que concuerda con un autoconcepto emocional bajo o inestable emocionalmente.

El modelo resultante de la regresión, por tanto, amplía aun más la importancia de las mismas y matiza alguno de los aspectos estudiados constituyendo así un avance sobre el estudio de esta enfermedad aportando a la vez datos de nuestro contexto.

\section{Conclusiones y líneas futuras de actuación}

Estos resultados, en su globalidad, no solo se han aportado para aproximar más el perfil psicológico de las personas con este trastorno, sino para dar a conocer al profesional de la Educación Física, al propio deportista y a cualquier persona interesada en la temática, las características básicas que pueden conducir al desarrollo de esta patología. De ésta manera, el presente estudio invita a reflexionar sobre la necesidad de proponer una educación apropiada para el buen uso del ejercicio físico y del deporte saludable en el periodo de formación del profesional deportivo, para intentar evitar la aparición de muchos de los trastornos en la imagen corporal.

Se han encontrado variables que actúan como predictoras de la vigorexia, que se podrían intentar modificar para el beneficio de los deportistas, como ansiedad, autoconcepto, horas semanales dedicadas a hacer ejercicio físico, etc.; las cuales podrían considerarse como un reto para los centros deportivos. De esta manera podrían asumir el papel de responsables de la formación deportiva, y de la actividad física como un medio saludable, con multitud de beneficios no solo físicos, sino metales, sociales, etc., y evitar ser acusados, de manera gratuita, de ser cómplices de este tipo de trastornos.

También podría ser una futura línea de investigación la puesta en marcha de programas de intervención para la modificación de conductas inadecuadas como ansiedad elevada (estado o rasgo) y baja autoestima, dada la importancia de estas variables en la aparición de vigorexia.

No queremos finalizar nuestra aportación sin reflexionar sobre la limitación en la generalización de los resultados del estudio a otros contextos distintos al nuestro realizando estudios comparativos entre deportistas de musculación y deportistas de cualquier otra modalidad, para analizar la existencia de diferencias en las puntuaciones de vigorexia obtenidas en ambos grupos. Sin duda, en el futuro será preciso abordar trabajos de tipo longitudinal, que permitan recoger información en distintos momentos y de esta manera poder comprobar la evolución de estas conductas de riesgo.

\section{Referencias}

1. Baile, J. I. (2005). Vigorexia cómo reconocerla y evitarla. Madrid: Síntesis.

2. Baile, J. I., Monroy, K.E. y Garay, F. (2005). Alteración de la imagen corporal en un grupo de usuarios de gimnasios. Enseñanza e Investigación en Psicología, 10(1), 161-169.

3. Candel, N., Olmedilla, A. y Blas, A. (2008). Relación entre la práctica de actividad física y el autoconcepto, la ansiedad y la depresión en chicas adolescentes. Cuadernos de Psicología del Deporte, 8 (1), 61-77.

4. Chi-Fu, J., Yang J., P.G. y Pope, H G. Jr. (2005). Male Body Image in Taiwan Versus the West: Yanggang Zhiqi Meets the Adonis Complex. American Journal of Psychiatry, 162(1), 263-269.

5. Contreras, O.R., Fernández, J.G., García, L.M., Palau, P. y Ponseti, J.
(2010). El Autoconcepto Físico y su relación con la práctica deportiva en estudiantes adolescentes. Revista de Psicología del Deporte, 19(1), 23-39.

6. De la Serna, I. (2004). Alteraciones de la imagen corporal: Anorexia, Vigorexia, Bulimia, Dismorfofobia y cuadros relacionados. Monografías de Psiquiatría, 2(16), 32-40.

7. Esnaola, I., Goñi, A. y Madariaga, J.M. (2008). El autoconcepto perspectivas de la investigación. Revista de Psicodidáctica, 13(1), 179-194.

8. Fanjul-Peyró, C. (2008). Modelos masculinos predominantes en el mensaje publicitario y su influencia social en la psicopatología del siglo XXI: La vigorexia. Revista Prismasocial, 1, 1-26.

9. Gallo, C., González, C., Salinero J.J. (2010). Comparison of the moti- 
vations, perceptions and futures expectations between physical activity and sport's science students of Camilo José Cela (Spain) and Hertfordshire University (England). Journal of Sport and Health Research, 2(3), 253-260.

10. García F. y Musitu G. (1999). Autoconcepto Forma 5. Madrid: TEA.

11. Garner, D.M. y Garfinkel, P.E. (1981). Body image in anorexia nervosa: measurement, theory and clinical implications. International Journal of Psychiatric in Medicine, 3, 263-284.

12. Goñi, A., Rodríguez, A. y Esnaola, I. (2010). Las autopercepciones físicas en la edad adulta y en la vejez. Psicothema, 22(3), 460-467.

13. Infante, G. y Goñi, E. (2009). Actividad físico-deportiva y autoconcepto físico en la edad adulta. Revista de Psicodidáctica, 14(1), 49-62.

14. Jeffrey, C.F., Gray, P., y Pope, H.G (2005). Male Body Image in Taiwan Versus the West: Yanggang Zhiqi Meets the Adonis Complex. American Journal Psychiatry, 162, 263-269.

15. Jenaro, C., Flores, N., Bermejo, B. y Cruz, M. (2011). Body Image Questionnaire for early detection of eating disorders. Acción Psicológica, 8(1), 7-20.

16. Jódar, I.M., Vives, L. y Garcés, E.J. (2002). Evaluación de los beneficios psicológicos del ejercicio Físico: evaluación de la ansiedad y de la depresión en la población de mujeres y su relación con la práctica de la actividad deportiva. II Congreso de Ciencias del Deporte, Universidad de Extremadura.

17. Lanzt, C.D., Rhea, D.J. y Cornelius, A.E. (2002). Muscle Dysmorphia in elite-level power lifters and body builders: a test of differences whiting a conceptual model. Journal of Strength and Conditioning Research, 16, 649-655.

18. López-Ibor, J.J. (Dtor.) (2009). DSM-IV-TR: Manual de diagnostico y estadístico de los trastornos mentales. Barcelona: Elsevier Masson.

19. Martell, C.A. (2006). Vigorexia: enfermedad o adaptación. Revista Digital de Educación Física y Deportes, 11 (99). Artículo disponible en: http://www.efdeportes.com/efd99/vigorex.htm

20. Mirella, A. (2008). Cuando la actividad física se aleja de la salud. Buenos Aires: Asociación de Psicología del Deporte.

21. Moreno, J. A., Hernández, A. y González-Cutre, D. (2009). Completando la teoría de la autodeterminación con las metas sociales: un estudio sobre la diversión en Educación Física. Revista Mexicana de Psicología, 26(2), 213-222.

22. Moreno, J. A., Cervelló, E. y Moreno, R. (2008). Importancia de la práctica físico-deportiva y del género en el autoconcepto físico de los 9 a los 23 ańos. International Journal of Clinical and Health Psychology, 8(1), 171-183.
23. Muńoz, R., y Martínez, A. (2007). Ortorexia y Vigorexia: ¿Nuevos Trastornos de la Conducta Alimentaria? Trastornos de la Conducta Alimentaria, 5, 457-482.

24. Olmedilla, A., Andreu, M. D. (2002). Propuesta de intervención psicológica para el control de hábitos alimentarios en deportistas jóvenes. Cuadernos de Psicología del Deporte, 2 (2), 13-28.

25. Pacheco, K.Z., Serrano, I. y Echegaray, I. (2007). Obesidad: el reto de la invisibilidad en el siglo XXI. Revista Puertorriqueña de Psicología, 18, 82-106

26. Pérez, V., Nelly-Valencia, M., Rodríguez, G.M. y Gempeler R. J. (2007) Acerca de un caso de dismorfia muscular y abuso de esteroides. Revista Colombiana de Psiquiatría, 36(1). 154-164.

27. Pope, H.G. (2000). The Adonis Complex. New York: Free Press.

28. Pope, H.G y Katz, D.L. (1994). Psychiatric and medical effects of anabolic-androgenic steroids: a controlled study of 160 athletes. Archives General Psychiatry, 51, 375-382.

29. Pope, H.G, Katz, D.L. y Hudson, J.L. (1993). Anorexia nervosa and "reverse anorexia" among 108 male bodybuilders. Comprehensive Psychiatry, 34(6), 406-409.

30. Pope, H.G., Katherine, A. Phillips, M.D. y Olivardia, R. (2003). The Adonis Complex: The Secret Crisis of Male Body Obsession. American Journal Psychiatry, 158, 1947-1948.

31. Raich, R.M. (2000). Imagen corporal. Madrid: Pirámide.

32. Ramos, P., Pérez de Eularte, L., Liberal, S. y Latorre, M. (2003). La imagen corporal en relación con los TCA en adolescentes vascos de 12 a 18 ańos. Revista de Psicodidáctica, 15-16, 67-74.

33. Spielberger, C.D., Gorsuch, R.L y Lushene. R.E. (1970). Manual for the State-Trait. Anxiety Inventory. Palo Alto, CA: Consulting Psychologists Press.

34. Spielberger, C.D., Gorsuch, R.L. y Lushene. R.E. (1999). Test de Ansiedad rasgo y estado (STAI). Validación española. Madrid: TEA Ediciones.

35. Thompson, J.K. (1990). Body image disturbances: assessment and treatment. New York: Pergamon Press.

36. Vaz, F.J., Peńas, E.V. y Ramos, M.I. (1999). Body image dissatisfaction in bulimia nervosa and atypical bulimia nervosa. German Journal of Psychiatry, 2, 59-74.

37. Vives, L. y Garcés, E.J. (2003). Hacia un modelo teórico explicativo del Burnout en deportistas. EduPsykhé: Revista de psicología y psicopedagogía, 2(2), 221-242.

38. Zagalaz, M.L., Martínez, E. y Rodríguez, I. (2005). Anorexia nerviosa y práctica de ejercicio físico para perder peso. Apunts: Educación Física y Deportes, 82, 12-18. 